\title{
Experimental Measurement of Electrically and Thermally Operated Convection in Liquid Dielectric
}

\author{
Member Ryu-ichiro Ohyama (Tokai University) \\ Member Kiyoji Kaneko (Kyushu Tokai University)
}

Key words : Electrohydrodynamics, Convection, Liquid dielectric, Fluid flow measurement, Image processing

Liquid dielectric frequently forms a convection field when an electric field is applied. The electrohydrodynamic phenomenon is caused by various effects such as ion drag pressure, dielectrophoresis and others. Experimental observations of the flow pattern have been reported in a number of interesting papers ${ }^{(1)(2)}$. Since the fluid motion is unsteady driving by effect on charges created with the applied field, it makes the distribution of momentary flow velocities over wide flow fields difficult to measure ${ }^{(3)}$. The authors have been studied on an image processing method for measuring flow velocities of the electrically operated convection fields in liquid dielectrics ${ }^{(4)}$. An interesting phenomenon on the driving of fluid motion in the liquid dielectrics is not only the electrically operated convection but also thermally operated convention superposed on that from a viewpoint of energetics. This letter reports an original measurement of electrically and thermally operated convection in the liquid dielectrics.

Fig.1 shows the experimental apparatus, in which a glass rod heater of $10 \mathrm{~mm}$ diameter is installed in the bottom of transparent cylindrical oil tank (9.6 $\ell$ ), a brass rod electrode with a diameter of $5 \mathrm{~mm}$ is immersed in $50 \mathrm{cSt}$ silicon oil. The heater and the electrode fit to a device for forming a natural convection field and an electrohydrodynamic convection field, respectively. Surface temperature of the heater is adjusted to $73^{\circ} \mathrm{C}$. The gap distance between the heater and the electrode is $28 \mathrm{~mm}$. The convection fields are visualized by illuminated tracer particles with a laser sheet beam of $1 \mathrm{~mm}$ thick at the vertical plane along both the axes of the heater and the electrode. Fig. 2 shows a digital flow image captured by the CCD camera. The tracer particles suspended in the silicon oil is low density polyethylene particles (average particle diameter of $0.5 \mathrm{~mm}$ ) with a dielectric constant and a volume resistivity close to those of the oil. The particles are mixed into the silicon oil, $0.05 \%$ in weight. Although the particles do not trace the silicon oil flow in the strict sense, the particles with a specific gravity close to that of the silicon oil can create a flow pattern similar to that of the oil.

The image processing for measuring flow velocity vectors is based on a correlation technique concerning binary tracer images, in which the distributions of 2-dimensional flow velocity vectors are obtained from the tracer particles identified by two digital images of sequential flow. The particle identification is carried out by a correlation process related to the pattern recognition from the two digital images with time intervals. In this experiment, since the uncertain particle positions with field image signals of NTSC have been considered as 0.5 pixel, the measurement error of flow velocity vectors should be only few percent.

Fig. 3 shows the experimental results. (a) is a velocity vector distribution of the flow field when the electrode potential $\phi$ is 0 . The flow field has been formed by natural convection caused by heating. (b), (c) and (d) are flow

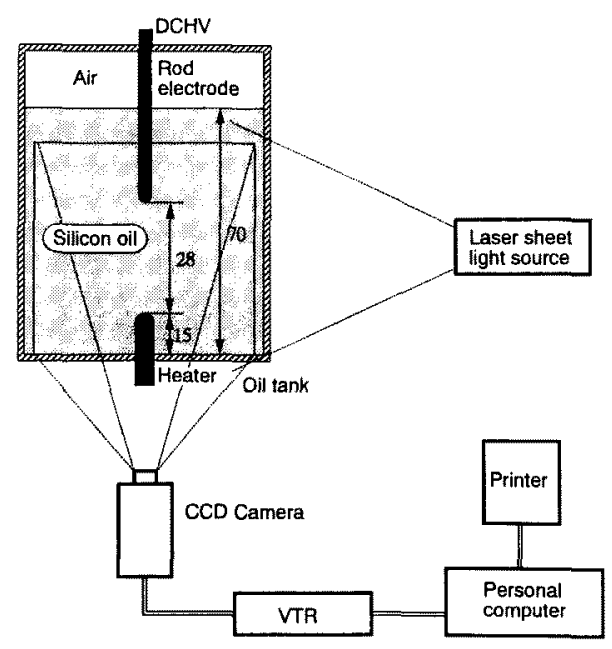

Fig.1 Experimental Apparatus. 


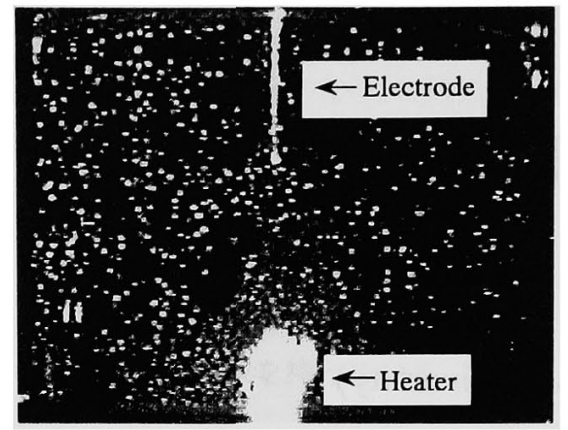

Fig.2 Visualization of convection field by illuminated particles.
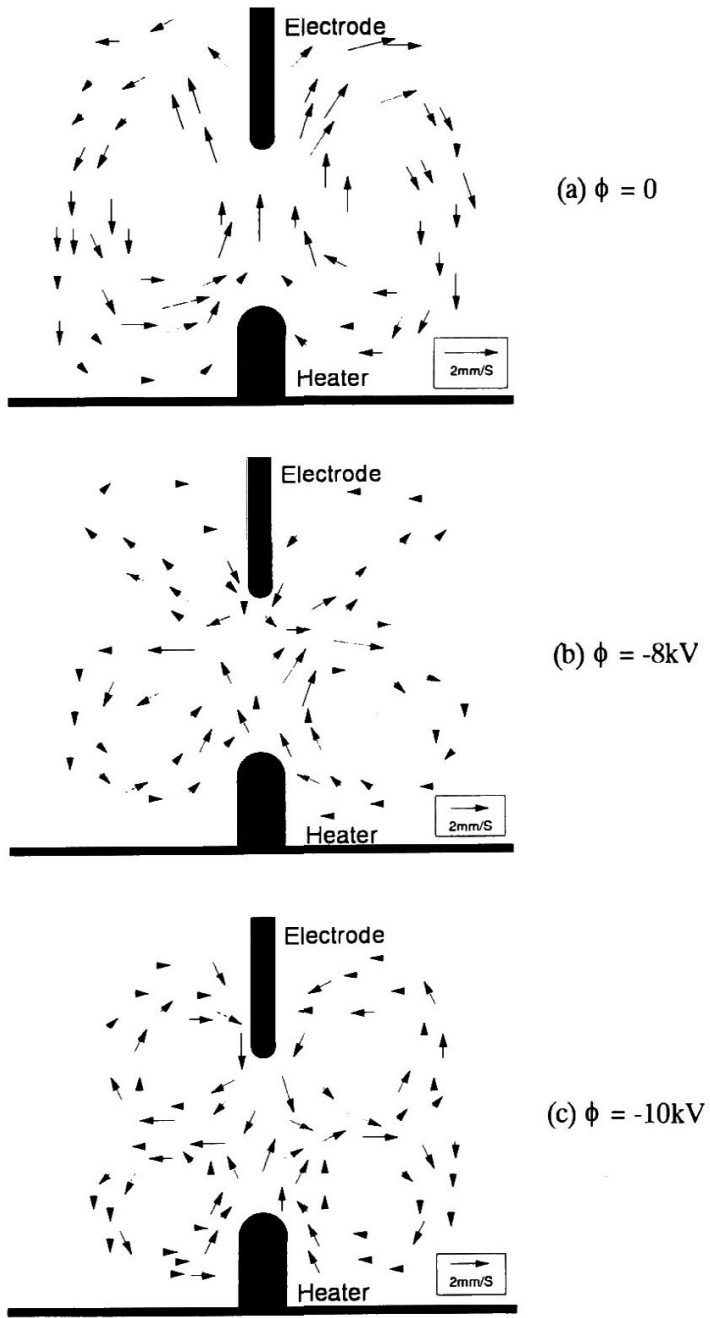

(c) $\phi=-10 \mathrm{kV}$

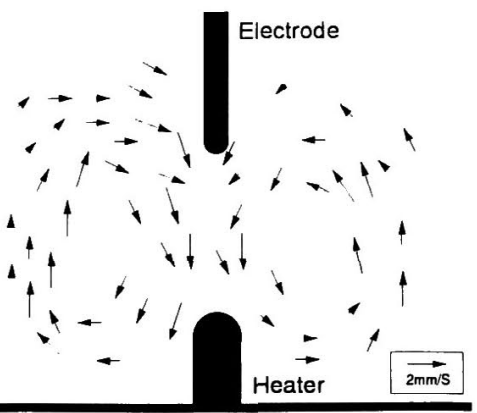

(d) $\phi=-12 \mathrm{kV}$

Fig.3 Experimental results obtained from image processing. fields mixed the natural convection with electrohydrodynamic convection when the electrode is biased with $-8 \mathrm{kV},-10 \mathrm{kV}$ and $-12 \mathrm{kV}$, respectively. These experimental results are momentary distributions of flow velocity vectors obtained from the convection fields. Reynolds number Re, which is obtained from the gap distance $28 \mathrm{~mm}$ and the maximum value of flow velocity along the axes of the heater and the electrode shown in Fig.3(d), and Grashoff number $\mathrm{Gr}$ are about 1.1 and 4.3 $\times 10^{3}$, respectively. It is seen from these results that the boundary between the natural convection and the electrohydrodynamic convection shifts to the heater in dependence on increased electrode potential. The electrohydrodynamic convection acts in the direction in which the buoyancy is negated. This is physically estimated that the convection is formed by a driving action causing energy rclcasc at the tip of electrode. An analysis of the convection fields requires more experiments including electric charge measurements for further examinations.

A part of this research has been supported by a research fund from the Kanagawa Academy of Science and Technology.

(Manuscript received Jul. 20. '95)

\section{References}

(1) W.H.Midendorf and G.H.Brown: AIEE Power Apparatus and System,Vol.77,pp.795-799 (1958)

(2) A.W.Mackey and J.C.Gibbings: Journal of Electrostatics, Vol.9, pp.355-366 (1981)

(3) R.Disselnkotter and K.Bamer: ibid., Vol.22,pp.43-60 (1989)

(4) T.Tsukiji, R.Ohyama and K.Kaneko: Trans. IEE Japan (in Japanese),Vol.112-A,No.9.pp.810-811 (1992)

Ryu-ichiro Ohy ama (Member) He was bom in Tokyo on Jan.

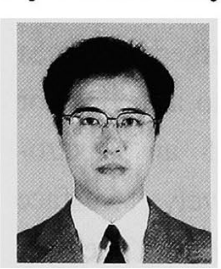
3,1964 . He received the D.E. degree from Tokai University in 1991. He is currently a Assistant Professor of Faculty of Engineering at Tokai University. His research interests include visualization and electro-hydrodynamics. $\mathrm{He}$ is a member of the institute of electrostatics and the visualization society of Japan.

Kiyoji Kaneko (Member) He was bom in Fukushima on Dec.

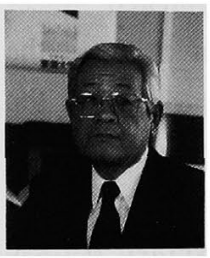
20, 1937. He received the M.E. degree from Tokyo Denki University in 1965 , and the D.E. degree from the Tokai University in 1987, respectively. In 1963 he joined Tokai University, and in 1994 moved to Kyushu Tokai University. He is currently a Professor and a dean of Faculty of Engineering at Kyushu Tokai University. His research interests include dielectrics and expert system of insulation deterioration problems. $\mathrm{He}$ is a member of the institute of electrostatics and the visualization society of Japan. 